\title{
RELAÇÕES BIDIMENSIONAIS E IDENTIDADES DA SEQUÊNCIA DE LEONARDO
}

\author{
Renata Passos Machado Vieira \\ Instituto Federal do Ceará - IFCE \\ re.passosm@gmail.com \\ Francisco Regis Vieira Alves \\ Instituto Federal do Ceará - IFCE \\ Paula Maria Catarino \\ Universidade de Trás-os-Montes e Alto Douro - UTAD \\ pcatarin@utad.pt
}

\section{Resumo}

Estudos relacionados à sequências lineares e recorrentes tem proporcionado a abordagem de outras bem menos conhecidas do que a Sequência de Fibonacci. Nesse sentido, este trabalho aborda uma breve introdução sobre a Sequência de Leonardo, assim como uma discussão à respeito das relações recorrentes bidimensionais desses números a partir do seu modelo unidimensional. Algumas propriedades e identidades são ainda introduzidas, de forma a estabelecer a relação existente entre essas duas sequências de números inteiros.

Palavras-chave: Relações bidimensionais; Relações de Recorrência; Sequência de Fibonacci; Sequência de Leonardo. de Leonardo.

\begin{abstract}
Studies related to linear and recurrent sequences have provided the approach to other that are less well known than the Fibonacci Sequence. In this sense, this paper deals with a brief introduction about the Leonardo Sequence, as well as a discussion about the two-dimensional recurring relations of this numbers from its one-dimensional model. Some properties and identities are further introduced in order to establish the existent relations between these two integer sequences.
\end{abstract}

Keywords: Two-dimensional relations; recurrence relations. Fibonacci Sequence. Leonardo Sequence. 


\section{Introdução}

Existe atualmente na literatura, um maior interesse pelo estudo de sequências lineares e recorrentes, bem como as suas aplicações no mundo científico. Uma sequência bastante estudada é a Sequência de Fibonacci, definida por:

$$
F_{n}=F_{n-1}+F_{n-2}, n \geqslant 2
$$

$\operatorname{com} F_{0}=0, F_{1}=1$.

Assim, os primeiros números de Fibonacci são descritos por:

$$
0,1,1,2,3,5,8,13,21,34,55,89,144,223,377,610,987,1597,2584,4181 .
$$

Sendo então apresentada em vários artigos de matemática, em que mostram o seu estudo em diversas áreas, tais como: cálculo, matemática aplicada, álgebra, estatística, física e informática, como pode-se encontrar em [1], [2], [4], [5], [6], [9], [10].

No trabalho de Catarino e Borges [7], é introduzida a Sequência de Leonardo, até então pouco conhecida e estudada na literatura. Assim, essa sequência é uma sequência linear e recorrente de segunda ordem, relacionando-a com a Sequência de Fibonacci, sendo então denotada por $L e_{n}$. Essa sequência é definida por:

$$
L e_{n}=L e_{n-1}+L e_{n-2}+1, n \geqslant 2
$$

$\operatorname{com} L e_{0}=L e_{1}=1$.

Assim, os primeiros números de Leonardo são descritos por:

$1,1,3,5,9,15,25,41,67,109,177,287,465,753,1219,1973,3193,5167,8361,13529,21891$.

A relação de recorrência (1.2) pode ser escrita de uma outra maneira, como faz Catarino e Borges [7]. Logo, a partir de $L e_{n}=L e_{n-1}+L e_{n-2}+1$ e $L e_{n+1}=L e_{n}+$ $L e_{n-1}+1$, pode-se então realizar a subtração entre essas duas equações, obtendo:

$$
\begin{aligned}
L e_{n}-L e_{n+1} & =L e_{n-1}+L e_{n-2}+1-\left(L e_{n}+L e_{n-1}+1\right) \\
L e_{n}-L e_{n+1} & =L e_{n-1}+L e_{n-2}+1-L e_{n}-L e_{n-1}-1 \\
L e_{n}-L e_{n+1} & =L e_{n-2}-L e_{n} \\
L e_{n+1} & =2 L e_{n}-L e_{n-2}, n \geqslant 2
\end{aligned}
$$

A relação entre a Sequência de Leonardo com a Sequência de Fibonacci é expressa pela seguinte proposição desenvolvida por Catarino e Borges [7]. 
Proposição 1.1. Para todo $n \geqslant 0$,

$$
L e_{n}=2 F_{n+1}-1 \text {. }
$$

Demonstração. A demonstração é feito por indução sobre $n$. Para $n=2$, tem-se que:

$$
\begin{aligned}
& L e_{n}=L e_{n-1}+L e_{n-2}+1, \\
& L e_{2}=L e_{1}+L e_{0}+1, \\
& L e_{2}=3 .
\end{aligned}
$$

Neste caso, a igualdade é válida. Supondo que (1.3) seja válida para algum $n=k, k \in \mathbb{N}$, tem-se que

$$
L e_{k}=L e_{k-1}+L e_{k-2}+1 \text {. }
$$

Se $n=k+1$, utilizamos as equações (1.2) e (1.1). Tem-se

$$
\begin{aligned}
L e_{k+1} & =L e_{k}+L e_{k-1}+1 \\
& =\left(2 F_{k+1}-1\right)+\left(2 F_{k}-1\right)+1 \\
& =2\left(F_{k+1}+F_{k}\right)-1 \\
& =2 F_{k+2}-1 .
\end{aligned}
$$

Obtemos da Proposição 1.1 que

$$
F_{n+1}=\frac{L e_{n}+1}{2} .
$$

Nas seções seguintes serão estudadas as relações recorrentes bidimensionais da Sequência de Leonardo, assim como algumas propriedades e identidades.

\section{Relações recorrentes bidimensionais de Leonardo}

De acordo com Harman [8], quando exploramos os números denotados por $L e(n, m)$ representamos os inteiros gaussianos $(n, m)=n+m i$, em que $n$ e $m$ são inteiros. Assim, para verificar esta extensão da sequência de Leonardo como sendo dimensão complexa, as relações recorrentes do caso bidimensional desta sequência serão explicadas no decorrer deste artigo.

Doravante, serão discutidas, algumas propriedades inerentes às relações bidimensionais oriundas da recorrência da Sequência de Leonardo

$$
L e(n+1)=2 L e(n)-L e(n-2) .
$$

ReviSeM, Ano 2019, No. 2, 156-173 
Definição 2.1. Os números da forma Le $(n, m)$ representarão os números da Sequência de Leonardo bidimensional e, devem satisfazer às seguintes condições bidimensionais de recorrência, em que $n, m \in \mathbb{N}$ :

$$
\left\{\begin{array}{l}
L e(n+1, m)=2 L e(n, m)-L e(n-2, m), \\
L e(n, m+1)=2 L e(n, m)-L e(n, m-2),
\end{array}\right.
$$

com os seguintes valores iniciais definidos: $\operatorname{Le}(0,0)=1, \operatorname{Le}(1,0)=1, \operatorname{Le}(0,1)=1+$ $i, \operatorname{Le}(1,1)=1+i$, em que $i^{2}=-1$ e $\operatorname{Le}(0)=\operatorname{Le}(1)=1, \operatorname{Le}(2)=3$.

Lema 2.2. Valem as seguintes propriedades:

(a) $\operatorname{Le}(n, 0)=\operatorname{Le}(n)$,

(b) $L e(0, m)=L e(m)+\left(\frac{L e(m-1)+1}{2}\right) i$,

(c) $\operatorname{Le}(n, 1)=\operatorname{Le}(n)+\left(\frac{\operatorname{Le}(n)+1}{2}\right) i$,

(d) $\operatorname{Le}(1, m)=\operatorname{Le}(m)+\left(\frac{\operatorname{Le}(m-1)+1}{2}\right) i$.

Demonstração. (a) Sendo $L e(n+1, m)=2 L e(n, m)-L e(n-2, m)$ e os valores iniciais $\operatorname{Le}(0,0)=1, \operatorname{Le}(1,0)=1$, aplicando o segundo princípio da indução finita sobre $n$, onde fixa-se $m=0$ e varia-se $n=0,1,2, \ldots$, observamos que

$$
\begin{aligned}
\operatorname{Le}(n, 0) & =2 \operatorname{Le}(n-1,0)-\operatorname{Le}(n-3,0) ; \\
L e(3,0) & =2 \operatorname{Le}(2,0)-\operatorname{Le}(0,0)=5=\operatorname{Le}(3) ; \\
\operatorname{Le}(4,0) & =2 \operatorname{Le}(3,0)-\operatorname{Le}(1,0)=9=\operatorname{Le}(4) ; \\
L e(5,0) & =2 \operatorname{Le}(4,0)-\operatorname{Le}(2,0)=15=\operatorname{Le}(5) ; \\
L e(6,0) & =2 \operatorname{Le}(5,0)-\operatorname{Le}(3,0)=25=\operatorname{Le}(6) ; \\
\vdots & \\
\operatorname{Le}(n-3,0) & =2 \operatorname{Le}(n-4,0)-\operatorname{Le}(n-6,0)=\operatorname{Le}(n-3) ; \\
\operatorname{Le}(n-2,0) & =2 \operatorname{Le}(n-3,0)-\operatorname{Le}(n-5,0)=\operatorname{Le}(n-2) ; \\
\operatorname{Le}(n-1,0) & =2 \operatorname{Le}(n-2,0)-\operatorname{Le}(n-4,0)=\operatorname{Le}(n-1) ; \\
\operatorname{Le}(n, 0) & =2 \operatorname{Le}(n-1,0)-\operatorname{Le}(n-3,0)=2 \operatorname{Le}(n-1)-\operatorname{Le}(n-3)=\operatorname{Le}(n) .
\end{aligned}
$$

Desse modo, verifica-se que vale a propriedade (a) $L e(n, 0)=L e(n)$.

De modo análogo, pode-se provar a validade de (b) $L e(0, m)=P(m)+P(m-1) i$, considerando a relação

$$
L e(n, m+1)=2 L e(n, m)-L e(n, m-2)
$$


para

$$
\operatorname{Le}(0,0)=1=\operatorname{Le}(0), \operatorname{Le}(1,0)=1=\operatorname{Le}(1), \operatorname{Le}(0,1)=1+i,
$$

verificando ainda a relação da Sequência de Leonardo com a Sequência de Fibonacci, onde $F(n)=F(n-1)+F(n-2)$ e $L e(n-2)=2 F(n+1)-1$ acarretam em $F(n+1)=$ $\frac{L e(n)+1}{2}$ (Proposição 1.1), sendo ainda os números iniciais de Fibonacci $F(0)=0, F(1)=$ $F(2)=1$. Analisando a recursividade para $n=0$ e variando $m=0,1,2,3, \ldots$, percebese que

$$
\begin{aligned}
L e(0, m) & =2 L e(0, m-1)-L e(0, m-3): \\
L e(0,3) & =2 L e(0,2)-L e(0,0)=2(3+i)-1=5+2 i=L e(3)+F(3) i \\
& =L e(3)+\left(\frac{L e(2)+1}{2}\right) i ; \\
L e(0,4) & =2 L e(0,3)-L e(0,1)=2(5+2 i)-(1+i)=9+3 i=L e(4)+F(4) i \\
& =L e(4)+\left(\frac{L e(3)+1}{2}\right) i ; \\
L e(0,5) & =2 L e(0,4)-L e(0,2)=2(9+3 i)-(3+i)=15+5 i=L e(5)+F(5) i \\
& =L e(5)+\left(\frac{L e(4)+1}{2}\right) i ; \\
L e(0,6) & =2 L e(0,5)-L e(0,3)=2(15+5 i)-(5+2 i)=25+8 i=L e(6)+F(6) i \\
& =L e(6)+\left(\frac{L e(5)+1}{2}\right) i ; \\
& \vdots \\
L e(0, m-3) & =2 L e(0, m-4)-L e(0, m-6)=L e(m-3)+F(m-3) i \\
& =L e(m-3)+\left(\frac{L e(m-4)+1}{2}\right) i \\
L e(0, m-1) & =2 L e(0, m-2)-L e(0, m-4)=L e(m-1)+F(m-1) i \\
& =L e(m-1)+\left(\frac{L e(m-2)+1}{2}\right) i \\
L e(0, m-2) & =2 L e(0, m-3)-L e(0, m-5)=L e(m-2)+F(m-2) i \\
& L e(m-2)+\left(\frac{L e(m-3)+1}{2}\right) i \\
& =L
\end{aligned}
$$




$$
\begin{aligned}
L e(0, m) & =2 \operatorname{Le}(0, m-1)-L e(0, m-3)=2\left[\operatorname{Le}(m-1)+\left(\frac{\operatorname{Le}(m-2)+1}{2}\right) i\right] \\
& -\left[\operatorname{Le}(m-3)+\left(\frac{\operatorname{Le}(m-4)+1}{2}\right) i\right]=\operatorname{Le}(m)+\left(\frac{\operatorname{Le}(m-1)+1}{2}\right) i .
\end{aligned}
$$

Validando a propriedade (b) $L e(0, m)=L e(m)+\left(\frac{L e(m-1)+1}{2}\right) i$.

Para demonstrar a propriedade (c), utiliza-se o mesmo princípio da indução, com $L e(n+1, m)=2 L e(n, m)-L e(n-2, m)$ e para os valores iniciais $L e(0)=L e(1)=$ $1, \operatorname{Le}(2)=3, \operatorname{Le}(0,1)=1+i, \operatorname{Le}(1,1)=1+i, \operatorname{Le}(2,1)=3+2 i$, onde pode-se estabelecer uma relação entre a Sequência de Leonardo e a Sequência de Fibonacci, em que $F(n)=$ $F(n-1)+F(n-2)$ e $L e(n-2)=2 F(n+1)-1$ acarretam novamente $F(n+1)=\frac{L e(n)+1}{2}$ (Proposição 1.1), sendo ainda os números iniciais de Fibonacci $F(0)=0, F(1)=F(2)=$ 1. Assim, fixando $m=1$ e variando $n=0,1,2,3, \ldots$, segue que

$$
\begin{aligned}
\operatorname{Le}(n, 1) & =2 \operatorname{Le}(n-1,1)-\operatorname{Le}(n-3,1) \\
\operatorname{Le}(3,1) & =2 \operatorname{Le}(2,1)-\operatorname{Le}(0,1)=2(3+2 i)-(1+i)=5+3 i=L e(3)+F(4) i \\
& =\operatorname{Le}(3)+\left(\frac{\operatorname{Le}(3)+1}{2}\right) i \\
\operatorname{Le}(4,1) & =2 \operatorname{Le}(3,1)-\operatorname{Le}(1,1)=2(5+3 i)-(1+i)=9+5 i=L e(4)+F(5) i \\
& =\operatorname{Le}(4)+\left(\frac{\operatorname{Le}(4)+1}{2}\right) i \\
\operatorname{Le}(5,1) & =2 \operatorname{Le}(4,1)-\operatorname{Le}(2,1)=2(9+5 i)-(3+2 i)=15+8 i=\operatorname{Le}(5)+F(6) i \\
& =\operatorname{Le}(5)+\left(\frac{\operatorname{Le}(5)+1}{2}\right) i \\
\operatorname{Le}(6,1) & =2 \operatorname{Le}(5,1)-\operatorname{Le}(3,1)=2(15+8 i)-(5+3 i)=25+13 i=\operatorname{Le}(6)+F(7) i \\
& =\operatorname{Le}(6)+\left(\frac{\operatorname{Le}(6)+1}{2}\right) i ; \\
& \vdots \\
\operatorname{Le}(n-3,1) & =2 \operatorname{Le}(n-4,1)-\operatorname{Le}(n-6,1)=\operatorname{Le}(n-3)+F(n-2) i \\
& =\operatorname{Le}(n-3)+\left(\frac{\operatorname{Le}(n-3)+1}{2}\right) i \\
\operatorname{Le}(n-2,1) & =2 \operatorname{Le}(n-3,1)-\operatorname{Le}(n-5,1)=\operatorname{Le}(n-2)+F(n-1) i
\end{aligned}
$$




$$
\begin{aligned}
& =L e(n-2)+\left(\frac{\operatorname{Le}(n-2)+1}{2}\right) i \\
\operatorname{Le}(n-1,1) & =2 L e(n-2,1)-\operatorname{Le}(n-4,1)=\operatorname{Le}(n-1)+F(n) i \\
& =\operatorname{Le}(n-1)+\left(\frac{\operatorname{Le}(n-1)+1}{2}\right) i \\
\operatorname{Le}(n, 1) & =2 \operatorname{Le}(n-1,1)-\operatorname{Le}(n-3,1) \\
& =2\left[\operatorname{Le}(n-1)+\left(\frac{\operatorname{Le}(n-1)+1}{2}\right) i\right]-\left[\operatorname{Le}(n-3)+\left(\frac{\operatorname{Le}(n-3)+1}{2}\right) i\right] \\
& =\operatorname{Le}(n)+\left(\frac{\operatorname{Le}(n)+1}{2}\right) i .
\end{aligned}
$$

Provando (c).

Finalizando a demonstração, tem-se que, de modo análogo, pode-se considerar a relação $\operatorname{Le}(n, m)=2 \operatorname{Le}(n, m-1)-\operatorname{Le}(n, m-3)$ e os valores $\operatorname{Le}(0)=\operatorname{Le}(1)=1, \operatorname{Le}(2)=$ $3, \operatorname{Le}(1,0)=1, \operatorname{Le}(1,1)=1+i, \operatorname{Le}(1,2)=3+i$, realizando a mesma relação entre as duas sequências, Leonardo e Fibonacci e, fixando $n=1$ e variando $m=0,1,2,3, \ldots$. Nota-se que:

$$
\begin{aligned}
\operatorname{Le}(1, m) & =2 L e(1, m-1)-L e(1, m-3): \\
\operatorname{Le}(1,3) & =2 \operatorname{Le}(1,2)-\operatorname{Le}(1,0)=2(3+i)-1=5+2 i=L e(3)+F(3) i \\
& =\operatorname{Le}(3)+\left(\frac{\operatorname{Le}(2)+1}{2}\right) i \\
\operatorname{Le}(1,4) & =2 \operatorname{Le}(1,3)-\operatorname{Le}(1,1)=2(5+2 i)-(1+i)=9+3 i=\operatorname{Le}(4)+F(4) i \\
& =\operatorname{Le}(4)+\left(\frac{\operatorname{Le}(3)+1}{2}\right) i \\
\operatorname{Le}(1,5) & =2 \operatorname{Le}(1,4)-\operatorname{Le}(1,2)=2(9+3 i)-(3+i)=15+5 i=\operatorname{Le}(5)+F(5) i \\
& =\operatorname{Le}(5)+\left(\frac{\operatorname{Le}(4)+1}{2}\right) i \\
\operatorname{Le}(1,6) & =2 \operatorname{Le}(1,5)-\operatorname{Le}(1,3)=2(15+5 i)-(5+2 i)=25+8 i=\operatorname{Le}(6)+F(6) i \\
& =\operatorname{Le}(6)+\left(\frac{\operatorname{Le}(5)+1}{2}\right) i
\end{aligned}
$$




$$
\begin{aligned}
\operatorname{Le}(1, m-3) & =2 \operatorname{Le}(1, m-4)-\operatorname{Le}(1, m-6)=\operatorname{Le}(m-3)+F(m-3) i \\
& =\operatorname{Le}(m-3)+\left(\frac{\operatorname{Le}(m-4)+1}{2}\right) i ; \\
\operatorname{Le}(1, m-2) & =2 \operatorname{Le}(1, m-3)-\operatorname{Le}(1, m-5)=\operatorname{Le}(m-2)+F(m-2) i \\
& =\operatorname{Le}(m-2)+\left(\frac{\operatorname{Le}(m-3)+1}{2}\right) i ; \\
\operatorname{Le}(1, m-1) & =2 \operatorname{Le}(1, m-2)-\operatorname{Le}(1, m-4)=\operatorname{Le}(m-1)+F(m-1) i \\
& =\operatorname{Le}(m-1)+\left(\frac{\operatorname{Le}(m-2)+1}{2}\right) i ; \\
\operatorname{Le}(1, m) & =2 \operatorname{Le}(1, m-1)-\operatorname{Le}(1, m-3) \\
& =2\left[\operatorname{Le}(m-1)+\left(\frac{\operatorname{Le}(m-2)+1}{2}\right) i\right]-\left[\operatorname{Le}(m-3)+\left(\frac{\operatorname{Le}(m-4)+1}{2}\right) i\right] \\
& =\operatorname{Le}(m)+\left(\frac{\operatorname{Le}(m-1)+1}{2}\right) i .
\end{aligned}
$$

Teorema 2.3. Para os dois inteiros, $n, m \in \mathbb{N}$, os números na forma $\operatorname{Le}(n, m)$ são descritos por:

$$
L e(n, m)=\left[L e(n) \cdot \frac{\operatorname{Le}(m)+1}{2}+\frac{L e(m)+1}{2}-1\right]+\frac{L e(n)+1}{2} \cdot \frac{L e(m-1)+1}{2} i .
$$

Demonstração. Fixando o número natural $n$, pode-se realizar a demonstração através de indução sobre $m$. Para o valor de $m=0$, utiliza-se as propriedades validadas anteriormente pelo Lema 2.2(b) e Lema 2.2(c), onde $L e(-1)=-1, \operatorname{Le}(0)=\operatorname{Le}(1)=1, \operatorname{Le}(2)=$ 3 , cujos valores iniciais são $\operatorname{Le}(0,0)=1, \operatorname{Le}(1,0)=1, \operatorname{Le}(0,1)=1+i, \operatorname{Le}(1,1)=1+i$. Para isso, serão calculados alguns valores de $L e(n, m)$, variando $m$.

Para $L e(n, 2)$, utiliza-se a recorrência $L e(n, m)=2 L e(n, m-1)-L e(n, m-4)$ com os valores iniciais $\operatorname{Le}(0,0)=1=\operatorname{Le}(0), \operatorname{Le}(1,0)=1=\operatorname{Le}(1), \operatorname{Le}(2,0)=3=$ $\operatorname{Le}(2), \operatorname{Le}(0,2)=3+i, \operatorname{Le}(1,2)=3+i, \operatorname{Le}(2,2)=7+2 i, \operatorname{com} m=2$ fixo e $n=$ $0,1,2,3, \ldots$, tem-se que:

$$
\begin{aligned}
\operatorname{Le}(n, 2) & =2 \operatorname{Le}(n-1,2)-\operatorname{Le}(n-3,2): \\
\operatorname{Le}(3,2) & =2 \operatorname{Le}(2,2)-\operatorname{Le}(0,2)=2(7+2 i)-(3+i)=11+3 i=(2 \operatorname{Le}(3)+1)+F(4) i \\
& =(2 \operatorname{Le}(3)+1)+\left(\frac{\operatorname{Le}(3)+1}{2}\right) i ;
\end{aligned}
$$


R. P. M. Vieira, F. R. V. Alves, P. M Catarino

$$
\begin{aligned}
& \operatorname{Le}(4,2)=2 \operatorname{Le}(3,2)-\operatorname{Le}(1,2)=2(11+3 i)-(3+i)=19+5 i=(2 \operatorname{Le}(4)+1)+F(5) i \\
& =(2 L e(4)+1)+\left(\frac{L e(4)+1}{2}\right) i \\
& \operatorname{Le}(5,2)=2 \operatorname{Le}(4,2)-\operatorname{Le}(2,2)=2(19+5 i)-(7+2 i)=31+8 i=(2 \operatorname{Le}(5)+1)+F(6) i \\
& =(2 L e(5)+1)+\left(\frac{L e(5)+1}{2}\right) i \\
& L e(6,2)=2 L e(5,2)-L e(3,2)=2(31+8 i)-(11+3 i)=51+13 i=(2 L e(6)+1)+F(7) i \\
& =(2 L e(6)+1)+\left(\frac{L e(6)+1}{2}\right) i \\
& \vdots \\
& \operatorname{Le}(n-3,2)=2 \operatorname{Le}(n-4,2)-\operatorname{LeP}(n-6,2)=(2 \operatorname{Le}(n-3)+1)+F(n-2) i \\
& =(2 L e(n-3)+1)+\left(\frac{L e(n-3)+1}{2}\right) i \\
& \operatorname{Le}(n-2,2)=2 \operatorname{Le}(n-3,2)-\operatorname{LeP}(n-5,2)=(2 \operatorname{Le}(n-2)+1)+F(n-1) i ; \\
& =(2 \operatorname{Le}(n-2)+1)+\left(\frac{\operatorname{Le}(n-2)+1}{2}\right) i \\
& \operatorname{Le}(n-1,2)=2 \operatorname{Le}(n-2,2)-\operatorname{LeP}(n-4,2)=(2 \operatorname{Le}(n-1)+1)+F(n) i ; \\
& =(2 \operatorname{Le}(n-1)+1)+\left(\frac{\operatorname{Le}(n-1)+1}{2}\right) i \\
& \operatorname{Le}(n, 2)=2 \operatorname{Le}(n-1,2)-\operatorname{Le}(n-3,2) \\
& =2\left[(2 \operatorname{Le}(n-1)+1)+\left(\frac{\operatorname{Le}(n-1)+1}{2}\right) i\right]-\left[(2 \operatorname{Le}(n-3)+1)+\left(\frac{\operatorname{Le}(n-3)+1}{2}\right) i\right] \\
& =(2 L e(n)+1)+\left(\frac{L e(n)+1}{2}\right) i .
\end{aligned}
$$

Fixando o valor de $m=3$, tem-se que:

$$
\begin{aligned}
\operatorname{Le}(n, 3) & =2 \operatorname{Le}(n-1,3)-\operatorname{Le}(n-3,3): \\
\operatorname{Le}(3,3) & =2 \operatorname{Le}(2,3)-\operatorname{Le}(0,3)=2(11+4 i)-(5+2 i)=17+6 i \\
& =(3 \operatorname{Le}(3)+2)+2 F(4) i \\
& =(3 \operatorname{Le}(3)+2)+2\left(\frac{\operatorname{Le}(3)+1}{2}\right) i ;
\end{aligned}
$$


R. P. M. Vieira, F. R. V. Alves, P. M Catarino

$$
\begin{aligned}
& \operatorname{Le}(4,3)=2 \operatorname{Le}(3,3)-\operatorname{Le}(1,3)=2(17+6 i)-(5+2 i)=29+10 i \\
& =(3 L e(4)+2)+2 F(5) i \\
& =(3 L e(4)+2)+2\left(\frac{L e(4)+1}{2}\right) i ; \\
& \operatorname{Le}(5,3)=2 \operatorname{Le}(4,3)-\operatorname{Le}(2,3)=2(29+10 i)-(11+4 i)=47+16 i \\
& =(3 L e(5)+2)+2 F(6) i \\
& =(3 L e(5)+2)+2\left(\frac{L e(5)+1}{2}\right) i ; \\
& \operatorname{Le}(6,3)=2 \operatorname{Le}(5,3)-\operatorname{Le}(3,3)=2(47+16 i)-(17+6 i)=77+16 i \\
& =(3 \operatorname{Le}(6)+2)+2 F(7) i \\
& =(3 L e(6)+2)+2\left(\frac{L e(6)+1}{2}\right) i \text {; } \\
& \vdots \\
& \operatorname{Le}(n-3,3)=2 \operatorname{Le}(n-4,3)-\operatorname{Le}(n-6,3)=(3 \operatorname{Le}(n-3)+2)+2 F(n-2) i \\
& =(3 L e(n-3)+2)+2\left(\frac{L e(n-3)+1}{2}\right) i ; \\
& \operatorname{Le}(n-2,3)=2 \operatorname{Le}(n-3,3)-\operatorname{Le}(n-5,3)=(3 \operatorname{Le}(n-2)+2)+2 F(n-1) i \\
& =(3 \operatorname{Le}(n-2)+2)+2\left(\frac{\operatorname{Le}(n-2)+1}{2}\right) i ; \\
& \operatorname{Le}(n-1,3)=2 \operatorname{Le}(n-2,3)-\operatorname{Le}(n-4,3)=(3 \operatorname{Le}(n-1)+2)+2 F(n) i \\
& =(3 \operatorname{Le}(n-1)+2)+2\left(\frac{\operatorname{Le}(n-1)+1}{2}\right) i ; \operatorname{Le}(n, 3) \\
& =2 \operatorname{Le}(n-1,3)-\operatorname{Le}(n-3,3) \\
& =\left[(3 \operatorname{Le}(n-1)+2)+2\left(\frac{\operatorname{Le}(n-1)+1}{2}\right) i\right]-[(3 \operatorname{Le}(n-3)+2) \\
& \left.+2\left(\frac{\operatorname{Le}(n-3)+1}{2}\right) i\right] \\
& =(3 L e(n)+2)+2\left(\frac{L e(n)+1}{2}\right) i .
\end{aligned}
$$

Reescrevendo as propriedades vistas no Lema 2.2 (a) e (b), com $L e(-1)=-1, \operatorname{Le}(0)=$ 
$L e(1)=1, L e(2)=3$, tem-se que:

$$
\begin{aligned}
\operatorname{Le}(n, 0) & =(\operatorname{Le}(n) F(1)+(F(1)-1))+\left(\frac{\operatorname{Le}(n)+1}{2}\right) F(0) i \\
& =\left[\operatorname{Le}(n)\left(\frac{\operatorname{Le}(0)+1}{2}\right)+\left(\frac{\operatorname{Le}(0)+1}{2}-1\right)\right]+\left(\frac{\operatorname{Le}(n)+1}{2}\right)\left(\frac{\operatorname{Le}(-1)+1}{2}\right) i \\
\operatorname{Le}(n, 1) & =(\operatorname{Le}(n) F(2)(F(2)-1))+\frac{\operatorname{Le}(n)+1}{2} \cdot F(1) i \\
& =\left[\operatorname{Le}(n) \cdot \frac{\operatorname{Le}(1)+1}{2}+\left(\frac{\operatorname{Le}(1)+1}{2}-1\right)\right]+\left(\frac{\operatorname{Le}(n)+1}{2}\right)\left(\frac{\operatorname{Le}(0)+1}{2}\right) i .
\end{aligned}
$$

Contudo, supondo para $m=1,2, \ldots, k-2$, sejam válidas as seguintes identidades descritas:

$$
\begin{aligned}
& L e(n, 0)=\left[L e(n) \cdot \frac{L e(0)+1}{2}+\left(\frac{L e(0)+1}{2}-1\right)\right]+\frac{L e(n)+1}{2} \cdot \frac{L e(-1)+1}{2} i ; \\
& L e(n, 1)=\left[L e(n) \cdot \frac{L e(1)+1}{2}+\left(\frac{L e(1)+1}{2}-1\right)\right]+\frac{L e(n)+1}{2} \cdot \frac{L e(0)+1}{2} i \\
& L e(n, 2)=\left[L e(n) \cdot \frac{L e(2)+1}{2}+\left(\frac{L e(2)+1}{2}-1\right)\right]+\frac{L e(n)+1}{2} \cdot \frac{L e(1)+1}{2} i ; \\
& L e(n, 3)=\left[L e(n) \cdot \frac{L e(3)+1}{2}+\left(\frac{L e(3)+1}{2}-1\right)\right]+\frac{L e(n)+1}{2} \cdot \frac{L e(2)+1}{2} i \text {; } \\
& L e(n, k-3)=\left[L e(n) \cdot \frac{L e(k-3)+1}{2}+\left(\frac{L e(k-3)+1}{2}-1\right)\right] \\
& +\frac{L e(n)+1}{2} \cdot \frac{L e(k-4)+1}{2} i \\
& L e(n, k-2)=\left[L e(n) \cdot \frac{L e(k-2)+1}{2}+\left(\frac{L e(k-2)+1}{2}-1\right)\right] \\
& +\frac{L e(n)+1}{2} \cdot \frac{L e(k-3)+1}{2} i \\
& L e(n, k-1)=\left[\operatorname{Le}(n) \cdot \frac{\operatorname{Le}(k-1)+1}{2}+\left(\frac{\operatorname{Le}(k-1)+1}{2}-1\right)\right] \\
& +\frac{L e(n)+1}{2} \cdot \frac{L e(k-2)+1}{2} i .
\end{aligned}
$$

Para $m=k$, a partir da recorrência $L e(n, k)=2 L e(n, k-1)-L e(n, k-3)$, tem-se 
que

$$
\begin{aligned}
\operatorname{Le}(n, k)= & 2 \operatorname{Le}(n, k-1)-\operatorname{Le}(n, k-3) \\
= & 2\left[\left(\operatorname{Le}(n)\left(\frac{\operatorname{Le}(k-1)+1}{2}\right)+\left(\left(\frac{\operatorname{Le}(k-1)+1}{2}\right)-1\right)\right)\right. \\
& \left.+\left(\frac{\operatorname{Le}(n)+1}{2}\right)\left(\frac{\operatorname{Le}(k-2)+1}{2}\right) i\right] \\
& -\left[\left(\operatorname{Le}(n)\left(\frac{\operatorname{Le}(k-3)+1}{2}\right)+\left(\left(\frac{\operatorname{Le}(k-3)+1}{2}\right)-1\right)\right)\right. \\
& \left.+\left(\frac{\operatorname{Le}(n)+1}{2}\right)\left(\frac{\operatorname{Le}(k-4)+1}{2}\right) i\right] \\
= & {\left[\operatorname{Le}(n)\left(\frac{\operatorname{Le}(k)+1}{2}\right)+\left(\left(\frac{L e(k)+1}{2}\right)-1\right)\right] } \\
& +\left(\frac{\operatorname{Le}(n)+1}{2}\right)\left(\frac{\operatorname{Le}(k-1)+1}{2}\right) i .
\end{aligned}
$$

Realizando operações algébricas, pode-se reduzir o Teorema 2.3 à

$$
\begin{aligned}
\operatorname{Le}(n, m) & =\left[\operatorname{Le}(n)\left(\frac{\operatorname{Le}(m)+1}{2}\right)+\left(\left(\frac{\operatorname{Le}(m)+1}{2}\right)-1\right)\right]+\left(\frac{\operatorname{Le}(n)+1}{2}\right)\left(\frac{\operatorname{Le}(m-1)+1}{2}\right) i \\
& =\left(\frac{\operatorname{Le}(m)+1}{2}\right)(\operatorname{Le}(n)+1)-1+(\operatorname{Le}(n)+1)\left(\frac{\operatorname{Le}(m-1)+1}{4}\right) i \\
& =(\operatorname{Le}(n)+1)\left(\frac{\operatorname{Le}(m)+1}{2}+i \frac{\operatorname{Le}(m-1)+1}{4}\right)-1 .
\end{aligned}
$$

Corolário 2.4. Uma outra abordagem do Teorema 2.3 é através da função da sequência de Fibonacci:

$$
L e(n, m)=F(n+1)(2 F(m+1)+i F(m))-1
$$

Demonstração. Utilizando a Proposição 1.1, tem-se que:

$$
\begin{aligned}
\operatorname{Le}(n, m) & =\left[\operatorname{Le}(n)\left(\frac{\operatorname{Le}(m)+1}{2}\right)+\left(\left(\frac{\operatorname{Le}(m)+1}{2}\right)-1\right)\right]+\left(\frac{\operatorname{Le}(n)+1}{2}\right)\left(\frac{\operatorname{Le}(m-1)+1}{2}\right) i \\
& =[(2 F(n+1)-1)(F(m+1))+(F(m+1)-1)]+F(n+1) F(m) i \\
& =[(F(m+1))(2 F(n+1)-1+1)-1]+F(n+1) F(m) i \\
& =[(F(m+1))(2 F(n+1))-1]+F(n+1) F(m) i \\
& =F(n+1)(2 F(m+1)+i F(m))-1 .
\end{aligned}
$$




\section{Algumas Identidades da Sequência de Leonardo na forma complexa}

Doravante, serão exploradas algumas identidades bidimensionais dessa sequência, relacionando-a com os números de Fibonacci.

Identidade 3.1. A soma dos números $L e(n, m)$ de índice $n$ ímpar pode ser descrita por

$$
\begin{aligned}
\sum_{l=1}^{n} \operatorname{Le}(2 l+1, m) & =\operatorname{Le}(2 n+2, m)-\operatorname{Le}(2, m)-n \\
& =(\operatorname{Le}(2 n+1)-3)\left[\left(\frac{L e(m)+1}{2}\right)+i\left(\frac{L e(m-1)+1}{4}\right)\right]-n .
\end{aligned}
$$

Demonstração. Utilizando que $L e(n+1, m)=L e(n, m)+L e(n-1, m)+1$ acarreta $\operatorname{Le}(n, m)=\operatorname{Le}(n+1, m)-\operatorname{Le}(n-1, m)-1$, pode-se verificar

$$
\begin{aligned}
L e(3, m) & =L e(4, m)-L e(2, m)-1 \\
L e(5, m) & =L e(6, m)-L e(4, m)-1 \\
L e(7, m) & =L e(6, m)-L e(6, m)-1 \\
\vdots & \\
L e(2 n-1, m) & =\operatorname{Le}(2 n, m)-L e(2 n-2, m)-1 \\
L e(2 n+1, m) & =\operatorname{Le}(2 n+2, m)-L e(2 n, m)-1
\end{aligned}
$$

Realizando a soma telescópica e usando o Teorema 2.3, tem-se que

$$
\begin{array}{rlrl}
\sum_{l=1}^{n} \operatorname{Le}(2 l+1, m) & & \operatorname{Le}(2 n+2, m)-\operatorname{Le}(2, m)-n \\
= & {\left[L e(2 n+2)\left(\frac{L e(m)+1}{2}\right)+\left(\left(\frac{L e(m)+1}{2}\right)-1\right)\right]} \\
& +\left(\frac{L e(2 n+2)+1}{2}\right)\left(\frac{L e(m-1)+1}{2}\right) i \\
& -\left[\left(L e(2)\left(\frac{L e(m)+1}{2}\right)+\left(\left(\frac{L e(m)+1}{2}\right)-1\right)\right]\right. \\
& -\left(\frac{L e(2)+1}{2}\right)\left(\frac{L e(m-1)+1}{2}\right) i-n
\end{array}
$$




$$
\begin{aligned}
= & {\left[L e(2 n+2)\left(\frac{L e(m)+1}{2}\right)+\left(\left(\frac{L e(m)+1}{2}\right)-1\right)\right] } \\
& +\left(\frac{L e(2 n+2)+1}{2}\right)\left(\frac{L e(m-1)+1}{2}\right) i \\
& -\left[\left(3\left(\frac{L e(m)+1}{2}\right)+\left(\left(\frac{L e(m)+1}{2}\right)-1\right)\right]-2\left(\frac{L e(m-1)+1}{2}\right) i-n\right. \\
= & {\left[\left(\frac{\operatorname{Le}(m)+1}{2}\right)(L e(2 n+2)-3)\right] } \\
& +i\left(\frac{L e(m-1)+1}{2}\right)\left(\frac{L e(2 n+2)+1}{2}-2\right)-n \\
= & {\left[\left(\frac{L e(m)+1}{2}\right)(L e(2 n+2)-3)\right] } \\
& +i\left(\frac{L e(m-1)+1}{4}\right)(\operatorname{Le}(2 n+2)-3)-n \\
= & (L e(2 n+2)-3)\left[\left(\frac{L e(m)+1}{2}\right)+i\left(\frac{L e(m-1)+1}{4}\right)\right]-n .
\end{aligned}
$$

Reescrevendo ainda essa propriedade em função da Sequência de Fibonacci, pela Proposição 1.1, tem-se que

$$
\begin{aligned}
\sum_{l=1}^{n} L e(2 l+1, m) & =L e(2 n+2, m)-L e(2, m)-n \\
& =(2 F(2 n+3)-4)\left(F(m+1)+i \frac{F(m)}{2}\right)-n .
\end{aligned}
$$

Identidade 3.2. A soma dos números $L e(n, m)$ de índice $n$ par e não nulo pode ser descrita por:

$$
\begin{aligned}
\sum_{l=1}^{n} L e(2 l, m) & =L e(2 n+1, m)-L e(1, m)-n \\
& =(L e(2 n+1)-1)\left[\frac{\operatorname{Le}(m)+1}{2}+i\left(\frac{L e(m-1)+1}{4}\right)\right]-n
\end{aligned}
$$


R. P. M. Vieira, F. R. V. Alves, P. M Catarino

Demonstração. De modo análogo à demonstração anterior, tem-se que

$$
\begin{aligned}
\operatorname{Le}(2, m) & =\operatorname{Le}(3, m)-\operatorname{Le}(1, m)-1 \\
\operatorname{Le}(4, m) & =\operatorname{Le}(5, m)-\operatorname{Le}(3, m)-1 \\
\operatorname{Le}(6, m) & =\operatorname{Le}(7, m)-\operatorname{Le}(5, m)-1 \\
\vdots & \\
\operatorname{Le}(2 n, m) & =\operatorname{Le}(2 n+1, m)-\operatorname{Le}(2 n-1, m)-1
\end{aligned}
$$

Realizando a soma telescópica e o Teorema 2.3:

$$
\begin{aligned}
& \sum_{l=1}^{n} L e(2 l, m)=L e(2 n+1, m)-L e(1, m)-n \\
& =\left[L e(2 n+1)\left(\frac{L e(m)+1}{2}\right)+\left(\left(\frac{L e(m)+1}{2}\right)-1\right)\right] \\
& +\left(\frac{\operatorname{Le}(2 n+1)+1}{2}\right)\left(\frac{\operatorname{Le}(m-1)+1}{2}\right) i \\
& -\left[\operatorname{Le}(1)\left(\frac{\operatorname{Le}(m)+1}{2}\right)+\left(\left(\frac{\operatorname{Le}(m)+1}{2}\right)-1\right)\right] \\
& -\left(\frac{L e(1)+1}{2}\right)\left(\frac{L e(m-1)+1}{2}\right) i-n \\
& =\left[\operatorname{Le}(2 n+1)\left(\frac{L e(m)+1}{2}\right)+\left(\left(\frac{L e(m)+1}{2}\right)-1\right)\right] \\
& +\left(\frac{\operatorname{Le}(2 n+1)+1}{2}\right)\left(\frac{\operatorname{Le}(m-1)+1}{2}\right) i \\
& -\left[\left(\frac{L e(m)+1}{2}\right)+\left(\left(\frac{L e(m)+1}{2}\right)-1\right)\right]-\left(\frac{L e(m-1)+1}{2}\right) i-n \\
& =\left(\frac{\operatorname{Le}(m)+1}{2}\right)(\operatorname{Le}(2 n+1)-1)+i\left(\frac{\operatorname{Le}(m-1)+1}{2}\right)\left(\frac{L e(2 n+1)+1}{2}-1\right)-n \\
& =\left(\frac{L e(m)+1}{2}\right)(\operatorname{Le}(2 n+1)-1)+i\left(\frac{\operatorname{Le}(m-1)+1}{4}\right)(L e(2 n+1)-1)-n \\
& =(L e(2 n+1)-1)\left[\frac{L e(m)+1}{2}+i\left(\frac{L e(m-1)+1}{4}\right)\right]-n
\end{aligned}
$$


Ou ainda, escrevendo em função da Sequência de Fibonacci, pela Proposição 1.1,

$$
\begin{aligned}
\sum_{l=1}^{n} L e(2 l, m) & =L e(2 n+1, m)-L e(1, m)-n \\
& =(L e(2 n+1)-1)\left[\frac{L e(m)+1}{2}+i\left(\frac{L e(m-1)+1}{4}\right)\right]-n \\
& =(2 F(2 n+2)-2)\left(F(m+1)+i \frac{F(m)}{2}\right)-n .
\end{aligned}
$$

Identidade 3.3. A soma dos $n$ primeiros números $L e(n, m)$, com índice $n$ maior que zero, pode ser descrita por:

$$
\begin{aligned}
\sum_{l=1}^{n} L e(l, m) & =L e(n+1, m)-L e(0, m)-L e(1, m)-n \\
& =(L e(n+1)-3)\left[\left(\frac{L e(m)+1}{2}\right)+i\left(\frac{L e(m-1)+1}{4}\right)\right]-n+1 .
\end{aligned}
$$

Demonstração. Similar às demonstrações anteriores, tem-se que

$$
\begin{aligned}
\operatorname{Le}(1, m) & =\operatorname{Le}(2, m)-\operatorname{Le}(0, m)-1 \\
\operatorname{Le}(2, m) & =\operatorname{Le}(3, m)-\operatorname{Le}(1, m)-1 \\
\operatorname{Le}(3, m) & =\operatorname{Le}(4, m)-\operatorname{Le}(2, m)-1 \\
\operatorname{Le}(4, m) & =\operatorname{Le}(5, m)-\operatorname{Le}(3, m)-1 \\
\vdots & \\
\operatorname{Le}(n, m) & =\operatorname{Le}(n+1, m)-\operatorname{Le}(n-1, m)-1
\end{aligned}
$$

Assim, através da soma telescópica e do Teorema 2.3, é fácil ver que

$$
\begin{aligned}
& \sum_{l=1}^{n} \operatorname{Le}(l, m) \\
= & L e(n+1, m)-\operatorname{Le}(0, m)-\operatorname{Le}(1, m)-n \\
= & {\left[\operatorname{Le}(n+1)\left(\frac{L e(m)+1}{2}\right)+\left(\left(\frac{L e(m)+1}{2}\right)-1\right)\right]+\left(\frac{L e(n+1)+1}{2}\right)\left(\frac{L e(m-1)+1}{2}\right) i } \\
& -\left[\operatorname{Le}(0)\left(\frac{L e(m)+1}{2}\right)+\left(\left(\frac{L e(m)+1}{2}\right)-1\right)\right]-\left(\frac{L e(0)+1}{2}\right)\left(\frac{L e(m-1)+1}{2}\right) i-n \\
& -\left[\operatorname{Le}(1)\left(\frac{\operatorname{Le}(m)+1}{2}\right)+\left(\left(\frac{L e(m)+1}{2}\right)-1\right)\right]-\left(\frac{L e(1)+1}{2}\right)\left(\frac{L e(m-1)+1}{2}\right) i-n
\end{aligned}
$$




$$
\begin{aligned}
= & {\left[L e(n+1)\left(\frac{L e(m)+1}{2}\right)+\left(\left(\frac{L e(m)+1}{2}\right)-1\right)\right]+\left(\frac{L e(n+1)+1}{2}\right)\left(\frac{L e(m-1)+1}{2}\right) i } \\
& -\left[\left(\frac{L e(m)+1}{2}\right)+\left(\left(\frac{L e(m)+1}{2}\right)-1\right)\right]-\left(\frac{L e(m-1)+1}{2}\right) i-n \\
& -\left[\left(\frac{L e(m)+1}{2}\right)+\left(\left(\frac{L e(m)+1}{2}\right)-1\right)\right]-\left(\frac{L e(m-1)+1}{2}\right) i-n \\
= & {\left[\left(\frac{L e(m)+1}{2}\right)(L e(n+1)-2)-\left(\frac{L e(m)+1}{2}\right)+1\right] } \\
& +i\left(\frac{L e(m-1)+1}{2}\right)\left[\left(\frac{L e(n+1)+1}{2}\right)-2\right]-n \\
= & {\left[\left(\frac{L e(m)+1}{2}\right)(\operatorname{Le}(n+1)-3)+1\right]+i\left(\frac{L e(m-1)+1}{2}\right)\left(\frac{L e(n+1)-3}{2}\right)-n } \\
= & {\left[\left(\frac{\operatorname{Le}(m)+1}{2}\right)(\operatorname{Le}(n+1)-3)+1\right]+i\left(\frac{L e(m-1)+1}{4}\right)(\operatorname{Le}(n+1)-3)-n } \\
= & (\operatorname{Le}(n+1)-3)\left[\left(\frac{L e(m)+1}{2}\right)+i\left(\frac{L e(m-1)+1}{4}\right)\right]-n+1 .
\end{aligned}
$$

Escrevendo essa identidade em função da Sequência de Fibonacci, pela Proposição 1.1, tem-se que

$$
\begin{aligned}
\sum_{l=1}^{n} L e(l, m) & =L e(n+1, m)-L e(0, m)-L e(1, m)-n \\
& =(L e(n+1)-3)\left[\left(\frac{L e(m)+1}{2}\right)+i\left(\frac{L e(m-1)+1}{4}\right)\right]-n+1 \\
& =(2 F(n+2)-4)\left[\left(F(m+1)+i\left(\frac{F(m)}{2}\right)\right]-n+1 .\right.
\end{aligned}
$$

\section{Conclusão}

Foi possível apresentar neste trabalho uma discussão referente ao processo de complexificação da Sequência de Leonardo, estabelecendo a sua relação com a Sequência de Fibonacci. Esse processo foi possível com a investigação em torno da inserção da unidade imaginária $i$ e o aumento dimensional das representações algébricas.

Vale salientar que o conteúdo relacionado à Sequência de Leonardo encontra-se escasso na literatura, sendo então estudada a partir do trabalho de Catarino e Borges [7], em que este trata da sua recorrência, fórmula de Binet e algumas identidades, estabelecendo ainda uma relação dessa sequência com a Sequência de Fibonacci. 
Assim, foram introduzidas, neste trabalho, as relações recorrentes bidimensionais de Leonardo e algumas identidades dessa sequência na forma complexa, a fim de compreender aspectos relevantes sobre a complexificação desses números utilizando propriedades matemáticas.

\section{Referências}

[1] M. Abrate et al., Colored compositions, invert operator and elegant compositions with the "black tie", Discrete Math. 335 (2014), 1-7.

[2] Z. Akyuz and S. Halici, Some identities deriving from the $n$th power of a special matrix, Adv. Difference Equ. 2012, 2012:223, 6 pp.

[3] E. G. S. Almeida, Propriedades e Generalizações dos Números de Fibonacci. Dissertação de Mestrado Profissional em Matemática em Rede Nacional PROFMATCCEN-UFPB, 2014.

[4] C. I. Amannah and N. D. Nanwin, The Existence of Fibonacci numbers in the algorithmic generator for combinatoric Pascal triangle. British Journal of Science, v. 11, n. 2, p. 62-84, 2014.

[5] K. T. Atanassov et al., New visual perspectives on Fibonacci numbers, World Scientific Publishing Co., Inc., River Edge, NJ, 2002.

[6] C. M. Campbell and P. P. Campbell, The Fibonacci length of certain centropolyhedral groups, J. Appl. Math. Comput. 19 (2005), no. 1-2, 231-240.

[7] P. M. M. C. Catarino and A. Borges, On Leonardo Numbers. Acta Mathematica Universitatis Comenianae (2019), 1-12.

[8] C. J. Harman, Complex Fibonacci numbers, Fibonacci Quart. 19 (1981), no. 1, 82-86.

[9] T. Koshy, Fibonacci and Lucas numbers with applications. Vol. 1, John Wiley \& Sons, Inc., NJ, 2001.

[10] R. P. M Vieira e F. R. V. Alves, Identidades Tribonacci, Revista Sergipana de Matemática e Educação Matemática, v. 4, no. 1, p. 216-226, 2019.

Submetido em 27 de Agosto de 2019. Aceito em 25 de Outubro de 2019. 